\title{
Recurrent, massive Kaposi's sarcoma pericardial effusion presenting without cutaneous lesions in an HIV infected adult: A Case Report
}

\author{
RODRICK KABANGILA ${ }^{1}$, WILLIAM MAHALU ${ }^{1}$, NESTORY MASALU ${ }^{1}$, HYASINTA JAKA $^{1}$ and \\ ROBERT N. PECK ${ }^{1,2^{*}}$ \\ ${ }^{1}$ Bugando University College of Health Sciences, Mwanza, Tanzania \\ ${ }^{2}$ Weill Cornell Medical College, New York, USA
}

\begin{abstract}
In this report we describe the case of a 22-year-old female who presented to our hospital with a 2 week history of chest tightness and easy fatigability. Examination and chest ultrasound revealed a massive pericardial effusion with evidence of tamponade. A rapid test for HIV was positive. Diagnostic and therapeutic pericardiocentesis was performed with good clinical response and revealed serosanguinous, exudative fluid. According to national guidelines, the patient was empirically treated for tuberculous pericarditis. Recurrence of the pericardial effusion occurred after 2 weeks and the cardiothoracic surgeons were consulted. Several days later, the patient was taken to the operating theatre and a pericardial window was performed with resultant drainage of over 5 litres of pericardial fluid. Visualization of the pericardium revealed a purple, multinodular mass of about $4 \times 6 \mathrm{~cm}$ on the epicardium consistent with Kaposi's sarcoma of the pericardium. Five litres of blood stained fluid were drained. Anti-tuberculosis treatment was stopped and the patient was referred to the oncology unit. The patient was started on antiretroviral treatment and Vincristine chemotherapy and the pericardial effusion resolved completely after 6 cycles of chemotherapy. Kaposi sarcoma should be considered as differential diagnosis in HIV/AIDS patient presenting with massive pericardial effusion.
\end{abstract}

Keywords: Kaposi's sarcoma, pericardial effusion, HIV, tamponade, vincristine, Tanzania

\section{Introduction}

Pericardial disease is common among HIV infected individuals worldwide with a reported average incidence of $21 \%$ according to several echocardiography and autopsy studies (Estok \& Wallach 1998). Most of this pericardial disease is mild and self limited but severe pericardial disease also occurs.

Over 185 cases of cardiac tamponade among HIV infected individuals have been reported in the literature (Gowda et al., 2003). In many of these cases, the cause of cardiac tamponade was never determined. Tuberculosis is the most commonly diagnosed cause of cardiac tamponade amongst HIV infected individuals, particularly in Africa (Cegielski et al., 1994; Gowda et al., 2003). Other commonly reported causes include bacterial infection, lymphoma and Kaposi's sarcoma (Gowda et al., 2003).

The diagnosis and therefore treatment of pericardial disease among HIV infected individuals remains difficult, particularly in resource limited settings. Due to the high prevalence of tuberculosis as a cause of pericardial effusion amongst HIV infected individuals, some have recommended that all large, persistent pericardial effusions be treated as tuberculous pericarditis in the absence of some obvious, other etiology Cegielski et al., 1994).

* Correspondence: Dr. Robert N. Peck; E-mail: rnp2002@gmail.com 
We report the presentation, workup and treatment of a 22-year-old HIV infected female who presented to Bugando Medical Centre in Mwanza, Tanzania, with a massive pericardial effusion and cardiac tamponade. In this report, we describe the presentation, workup, diagnosis and treatment of this patient who was found to have a Kaposi's sarcoma of the pericardium. We also review the literature related to HIV and Kaposi's sarcoma of the pericardium.

\section{Case Presentation}

Bugando Medical Centre (BMC) is an 850 bed referral hospital located in Mwanza city in northwestern Tanzania on the southern border of Lake Victoria. In 2009, a 22-year-old female was referred to our hospital with a diagnosis of cardiomyopathy. On review, the patient was found to have a history of chest pain on exertion, awareness of heart beat, chest tightness and easy fatigability for two weeks. On exam, the patient was afebrile with a pulse rate of 130 beats per minute, a blood pressure of 104/74 $\mathrm{mmHg}$ and a respiratory rate of 20 cycles per minute. No cutaneous or mucosal lesions were present. Multiple, approximately 1-2 cm firm, non-tender, mobile lymph nodes were found in the cervical, axillary and inguinal regions.

On cardiac examination, the apex beat was not palpable and the heart sounds were distant. The jugular vein was distended to the level of the ear with the patient sitting in the upright position. Tender hepatomegaly was also present and liver edge was palpable $6 \mathrm{~cm}$ below the right costal margin. Lower extremity pitting oedema was present to the level of the knees. The lungs were clear to auscultation.

The patient was admitted to the medical ward with a diagnosis of pericardial effusion. A complete blood count revealed a white blood cell count of 11.4 cells $/ \mathrm{mm} 3$ (with a differential of $69 \%$ neutrophils, $23 \%$ lymphocytes, $6 \%$ monocytes and $1 \%$ eosinophils), a haemoglobin of $8.9 \mathrm{~g} / \mathrm{dl}$ and a platelet count of 140 cells $/ \mathrm{mm} 3$. The ESR was $40 \mathrm{~mm} / \mathrm{hr}$. Renal and liver function tests were all within normal range. A rapid test for HIV was performed and was positive and the patients CD4 count was reported as 320 cells/ul.

Chest $x$-ray revealed an enlarged cardiac silhouette (cardiothoracic ratio 0.7 ) with a globular shape. Echocardiogram revealed massive pericardial effusion with right ventricular collapse. The echocardiogram was otherwise normal with no valvular dysfunction or pericardial masses.

A bedside therapeutic pericardiocentesis was performed and $400 \mathrm{ml}$ of serosanguinous fluid were removed with almost immediate clinical improvement. Two samples of the pericardial fluid were sent to the laboratory for biochemistry, microbiology and cytology. The total protein of the fluid was elevated at $60.6 \mathrm{~g} / \mathrm{l}$ but the glucose was normal $(7.1 \mathrm{mmol} / \mathrm{l})$. Gram staining and acid fast staining of the fluid were both negative and bacterial cultures revealed no growth. On cytology, one sample revealed acellular material but the other revealed "red blood cells and white blood cells in the same ratio as peripheral blood with no atypical cells."

On hospital day 3 the patient was empirically started on standard four drug antituberculous treatment and prednisone $40 \mathrm{mg}$ PO twice daily according to the national regimen for treatment of TB pericarditis. She remained on the medical ward for 7 days. On hospital day 7 the patient was discharged in good condition through a local HIV clinic to commence counselling for the initiation of antiretroviral therapy. One week later, 2 weeks 
after starting anti-TB chemotherapy, the patient was started on antiretroviral therapy with zidovudine, lamivudine and efaverenz at standard adult doses.

Two weeks after discharge, the patient returned to BMC with complaints of difficulty in breathing, generalized body malaise, night sweats and fevers as well as weight loss. On examination, the patient had mild dyspnoea. Her blood pressure was 117/64 $\mathrm{mmHg}$ and her pulse rate was 117. On cardiac examination, the apex beat was once again not palpable and the heart sounds were distant. A positive pulsus paradoxus and Ewart's sign were noted on examination.

Repeat chest x-ray was unchanged from the prior admission with and enlarged, globular cardiac silhouette but clear lungs. A repeat bedside chest ultrasound revealed recurrent massive pericardial effusion. A second therapeutic pericardiocentesis was performed and 1 litre of serosanguinous fluid was removed. Due to the recurrence of the patient's pericardial effusion and the apparent lack of response anti-tuberculosis therapy, the medical team consulted the hospitals cardiothoracic surgeon.

On hospital day 21, the patient was taken to the operating theatre. An anterolateral thoracotomy was made in the left fourth intercostals space. The parietal pleura was normal with $100 \mathrm{ml}$ of effusion. The pericardium bulged into the incision, obscuring the lung which was compressed posteriorly. The pericardium was punctured and $20 \mathrm{ml}$ of serous fluid were aspirated. A longitudinal incision was then made into the pericardial sac and a litre of serosanguinous fluid was sucked out of the sac. This left a redundant pericardium which was excised to create a window of $10 \mathrm{~cm}$ in diameter.

On visualization of the epicardium through the pericardial window, the surface of the ventricular apex was seen to be studded with a purple, hemorrhagic multinodular mass that measured approximately $3 \times 5 \mathrm{~cm}$ and was surrounded by bloody fluid. An attempt to biopsy these nodules found them to be very friable; easily bleeding and fixed to the epicardium. Thus biopsy was abandoned, an intrapleural drain was inserted and the thoracotomy was closed. The patient had an uneventful recovery from surgery. After draining 3 additional litres of serosanguinous fluid, the intrapleural drain output became minimal and the drain was removed on post-operative day 14.

Based on the clinical history and operative findings, the patient was diagnosed with Kaposi's sarcoma of the pericardium. Repeat examination of the skin and mucous membranes again revealed no mucocutaneous lesions. Anti-tuberculous therapy was stopped. After recovering from surgery, the patient was transferred to the hospital's oncology ward under the care of the hospital's oncologist.

On the oncology ward, the patient received 6 cycles of vincristine given in 2 weekly intervals at a dose of $1.4 \mathrm{mg} / \mathrm{m}^{2}$. Doxorubicin was withheld due to the pericardial involvement of the disease and the concern for cardiac toxicity. After a total of 6 cycles of chemotherapy, the patient reported complete resolution of her symptoms. A repeat chest $\mathrm{x}-$ ray revealed a normal cardiac silhouette. The patient was last seen 3 months after the completion of her chemotherapy and is doing well.

\section{Discussion}

In this report we describe the case of a 22 year old HIV infected female who presented to our hospital with massive, recurrent Kaposi's sarcoma (KS) pericardial effusion. This case illustrates many important points about pericardial KS in patients in HIV. 
Pericardial disease is very common among HIV infected individuals. According to 15 echocardiographic and autopsy studies, the average incidence of pericardial effusion in patients with HIV was 21\% (Estok \& Wallach, 1998). Most pericardial effusions in patients with HIV are mild but over 185 cases of cardiac tamponade due to massive or rapidly accumulating pericardial effusion have been reported in the literature (Gowda et al., 2003). In our patient, the pericardial effusion was both massive and recurrent. Despite several attempts to drain the pericardial effusion, cardiac tamponade recurred 3 times. At the time of pericardial window, over 5 litres of pericardial fluid were drained from the pericardial space.

The most common cause of pericardial effusion among HIV infected individuals is tuberculosis. According to several large case series of pericardial effusion among patients with HIV living in the United States, Mycobacterium tuberculosis was the most commonly identified cause of pericardial effusion and accounted for $15-25 \%$ of all pericardial effusions (Estok \& Wallach, 1998; Chen et al., 1999; Gowda et al., 2003). Tuberculosis is even more prevalent as a cause of pericardial effusion in sub Saharan Africa. In one study from Tanzania, 14/14 patients admitted to a referral hospital with HIV and massive pericardial effusion who underwent pericardiocentesis had tuberculous pericarditis (Cegielski et al. 1994). These results have led to the recommendation that all cases of massive pericardial effusion in HIV infected patients be treated as tuberculosis unless some other cause can easily be identified.

Of course, pericardial effusions can also be caused by many things other than tuberculosis. In several studies from the United State of patients with HIV and pericardial effusion the other leading causes were: non-tuberculous mycobacteria $(\approx 15 \%)$, bacterial $(\approx 10 \%)$, lymphoma $(\approx 5 \%)$ and KS $(\approx 5 \%)$. Other causes included Cryptococcus neoformans, Nocardia asteroides, Aspergillus species, cytomegalovirus, and herpes simplex. The cause of pericardial effusion was not determined in $\approx 20-40 \%$ of cases ((Estok \& Wallach, 1998; Chen et al., 1999). Very little data is available in the literature about non-tuberculous causes of pericardial effusion in sub Saharan Africa and more study is needed in this area.

Kaposi's sarcoma pericardial effusions can be massive and are often hemorrhagic, as in this case (Stotka et al., 1989; Chyu et al., 1998; Atar et al., 1999). In most cases, mucocutaneous KS lesions are present but there has been at least one other reported case of pericardial KS reporting without evidence of mucocutaneous KS (Chyu et al., 1998). For this reason, the absence of mucocutaneous KS should not exclude the possibility of KS as the cause of pericardial effusion.

The diagnosis of KS as a cause of pericardial effusion is difficult in the absence of surgical intervention. In 1 case from the United States, laboratory investigation of the pericardial fluid was negative except for the presence of red blood cells and the diagnosis of KS was only confirmed after autopsy (Stotka, Good et al., 1989). In at least one report, echocardiogram was used to visualize multinodular KS lesions on the apical epicardium (Chyu et al., 1998). Pericardial KS typically involves the epicardium (visceral pericardium) and not the parietal pericardium and can be only visualized after the opening of the parietal pericardium in surgery or autopsy.

Treatment of Kaposi's sarcoma pericardial effusion related to HIV infection involves a combination of drainage, anti-retroviral therapy and chemotherapy as in this case. Urgent drainage of KS pericardial effusions is indicated in any patient signs of cardiac tamponade. This case illustrates how KS pericardial effusions can be massive and can rapidly re-accumulate after pericardiocentesis. For this reason, some have recommended 
early surgical intervention in patients with massive KS pericardial effusions (Vijay et al., 1996). Antiretroviral therapy is recommended in all patients with KS, regardless of the CD4 count. Protease inhibitor based regimens and protease inhibitor regimens are equally efficacious in inducing resolution of KS (Martinez et al., 2006). Chemotherapy is indicated for patient with visceral KS as well as for some patients with severe or disfiguring cutaneous KS. Many chemotherapy regimens have been used for the treatment of KS but liposomal anthracyclines seem to be superior and are first-line in most developed countries (Lee \& Mitsuyasu, 1996; Aldenhoven et al., 2006). In some developing countries such as Tanzania, vincristine alone has been used as in this case due to the reasonable outcomes, low cost and availability of this regimen (Mlombe, 2008). At our centre, the vincristine would have been combined with doxorubicin if the pericardium had not been involved.

In conclusion, in this report we describe the case of a 22 year old female who presented to our referral hospital in shock with cardiac tamponade and was diagnosed with HIV and a massive pericardial effusion. The patient did well with rapid pericardiocentesis, pericardial window, chemotherapy and antiretroviral therapy. This case illustrates the difficulty of diagnosing KS pericardial effusion in resources limited settings but also the good outcomes that can be achieved with drainage, chemotherapy and antiretroviral therapy. More research is desperately regarding pericardial effusion among HIV infected individuals in resource-limited settings.

\section{Acknowledgements}

We would like to thank Dr. Charles Majinge, Director of Bugando Medical Centre, for his support.

\section{References}

Aldenhoven, M., Barlo, N. P. \& Sanders, C.J. (2006) Therapeutic strategies for epidemic Kaposi's sarcoma. International Journal of STD and AIDS 17, 571-578.

Atar, S., Chiu, J., Forrester, J.S., \& Siegel, R.J. (1999) Bloody pericardial effusion in patients with cardiac tamponade: is the cause cancerous, tuberculous, or iatrogenic in the 1990s? Chest 116, 1564-1569.

Cegielski, J. P., Lwakatare, J., Dukes, C.S., Lema, L.E., Lallinger, G.J., Kitinya, J., Reller, L.B., \& Sheriff, F. (1994) Tuberculous pericarditis in Tanzanian patients with and without HIV infection. Tubercle and Lung Disease 75, 429-434.

Chen, Y., Brennessel, D., Walters, J., Johnson, M., Rosner, F., \& Raza, M. (1999) Human immunodeficiency virus-associated pericardial effusion: report of 40 cases and review of the literature. American Heart Journal 137, 516-521.

Chyu, K.Y., Birnbaum, Y., Naqvi, T., Fishbein, M.C. \& Siegel, R.J. (1998) Echocardiographic detection of Kaposi's sarcoma causing cardiac tamponade in a patient with acquired immunodeficiency syndrome. Clinical Cardiology 21, 131-133.

Estok, L. \& Wallach, F. (1998) Cardiac tamponade in a patient with AIDS: a review of pericardial disease in patients with HIV infection. Mount Sinai Journal of Medicine 65, 33-39.

Gowda, R.M. \& Khan, I.A. (2003) Cardiac tamponade in patients with human immunodeficiency virus disease. Angiology 54, 469-474. 
Lee, F.C. \& Mitsuyasu, R.T. (1996) Chemotherapy of AIDS--related Kaposi's sarcoma. Hematology/Oncology Clinics of North America 10, 1051-1068.

Martinez, V., Caumes, E., Gambotti, L., Ittah, H., Morini, J.P., Deleuze, J., Gorin, I., Katiama, C., Bricaire, F., \& Dupin, N. (2006) Remission from Kaposi's sarcoma on HAART is associated with suppression of HIV replication and is independent of protease inhibitor therapy. British Journal of Cancer 94, 1000-1006.

Mlombe, Y. (2008) Management of HIV associated Kaposi's sarcoma in Malawi. Malawi Medical Journal 20, 129-132.

Stotka, J.L., Good, C.B., Downer, W.R. \& Kapoor, W.N. (1989) Pericardial effusion and tamponade due to Kaposi's sarcoma in acquired immunodeficiency syndrome. Chest 95, 1359-1361.

Vijay, V., Aloor, R.K., Yalla, S.M., Bebawi, M. \& Kashan, F. (1996) Pericardial tamponade from Kaposi's sarcoma: role of early pericardial window. American Heart Journal 132, 897-899. 